\title{
Escolaridade, trabalho, renda e saúde mental: um estudo retrospectivo e de associação com usuários de um Centro de Atenção Psicossocial
}

\author{
I ${ }^{1}$ Ioneide de Oliveira Campos, ${ }^{2}$ Daniel Marinho Cezar da Cruz, \\ ${ }^{3}$ Yasmim Bezerra Magalhães, ${ }^{4}$ Daniela da Silva Rodrigues I
}

Resumo: Estudos de perfil sociodemográfico são importantes para o planejamento e análise das necessidades de uma população. No campo da saúde mental, pesquisas sobre o perfil de usuários desses serviços ainda são incipientes no Brasil. Objetivou-se descrever o perfil sociodemográfico de usuários de um Centro de Atenção Psicossocial (CAPS) e verificar se existem associações entre a variável escolaridade com as demais variáveis sociodemográficas. Trata-se de estudo transversal descritivo e associativo, do tipo retrospectivo, com coleta a partir de prontuários, protocolo de acolhimento, relatório social e anamnese psiquiátrica. A análise de dados foi descritiva e com testes de associação. Identificou-se uma proporção usuários do sexo feminino no CAPS ( $\mathrm{n}=324)$. Os usuários eram oriundos majoritariamente da Regiāo Centro-Oeste do país ( $\mathrm{n}=239)$, seguida da Nordeste $(\mathrm{n}=144)$. Os resultados indicaram um alto número de desemprego ( $\mathrm{n}=130)$ e níveis elevados para baixa escolaridade $(\mathrm{n}=264)$. As variáveis: trabalho $(\mathrm{p}=0.0005)$, ocupação $(\mathrm{p}=0.0005)$, renda $(\mathrm{p}<0.0001)$, quantidade de filhos $(\mathrm{p}<0.007)$ e receber benefício $(\mathrm{p}=0.02)$ apresentaram correlaçóes positivas com o nível de escolaridade. Conclui-se que pesquisas de correlação entre dados sociodemográficos e indicadores da saúde mental podem fornecer evidências para a criação de políticas públicas nesse campo e desdobramentos para a inclusão social dos usuários.

> Palavras-chave: saúde mental; condições sociais; educação; trabalho; reabilitação.

\author{
${ }^{1}$ Universidade de Brasília. \\ Ceilândia-DF, Brasil \\ (ioncampos2016@gmail.com). \\ ORCID: 0000-0002-6803-2725 \\ ${ }^{2}$ Leeds Beckett University, West \\ Yorkshire, England (d.m.cezar-da- \\ cruz@leedsbeckett.ac.uk). \\ ORCID: 0000-0002-4708-354X \\ ${ }^{3}$ Universidade de Brasília. Asa \\ Norte-DF, Brasil (yasmimbma@ \\ gmail.com). \\ ORCID: 0000-0002-0614-5644 \\ ${ }^{4}$ Universidade de Brasília. \\ Ceilândia-DF, Brasil \\ (danirodrigues.to@gmail.com). \\ ORCID: 0000-0001-7391-1794
}

Recebido em: 04/09/2019

Aprovado em: 27/03/2020 Revisado em: 21/07/2021 


\section{Introdução}

As transformaçôes do modelo de assistência em saúde mental no Brasil, impulsionadas pelo processo de Reforma Psiquiátrica, implicaram a criação de serviços substitutivos de atenção à saúde mental. Estes permitiram discussóes sobre a rede de suporte social e de saúde, assim como demandas e necessidades de políticas públicas. Tais transformaçôes possibilitaram ativar diferentes dispositivos de tratamento e assistência para as pessoas em sofrimento psíquico (SARACENO, 1999).

Segundo consta nos documentos ministeriais, somente a partir da década de 1990, a formulação de políticas públicas de saúde mental impulsionou a implantação de serviços públicos a ela relacionados, por meio das Portarias nos 189/1991 e 224/1992, o que, para Pitta (2010), significou a reforma como política pública consentida. Caminhou-se, a partir do ano 2000, para a Reforma Legal, com a criação de um importante arcabouço normativo, de expansão da rede comunitária e do controle dos hospitais (PITTA, 2010). Em 2001, foi sancionada a Lei no 10.216 , afirmando os direitos das pessoas em sofrimento mental e redirecionando o modelo assistencial em saúde mental (BRASIL, 2013).

Na década de 2000, ampliou-se a rede de atenção psicossocial (Raps), que passou a integrar, a partir do Decreto Presidencial no 7508/2011, o conjunto das redes indispensáveis na constituição das regiōes de saúde (BRASIL, 2013). A inserção da assistência em saúde mental na atenção básica, como uma proposta de rede, vem sendo desenvolvida por meio de açóes que se relacionam aos princípios de integralidade da atenção, participação social, ampliação do conceito saúde-doença, interdisciplinaridade do cuidado e territorialização, evidenciando a existência de princípios norteadores comuns entre a reforma psiquiátrica e a reforma sanitária (BALLARIN; MIRANDA; FUENTES, 2010).

Os equipamentos substitutivos são Centros de Atenção Psicossocial (CAPS), os Centros de Convivência (CECOS), a Inclusão Social pelo Trabalho e as Residências Terapêuticas, que por meio do Programa de Volta para Casa tornaram possível a desinstitucionalização de moradores do hospital psiquiátrico (LUSSI et al., 2019). Desse modo, uma das formas de se conhecer as características dos usuários dos equipamentos substitutivos está na condução de análises sociodemográficas e epidemiológicas, por exemplo, tendo os CAPS, como cenário de pesquisas. Essas envolvem variáveis complexas, principalmente quando associadas às econômicas e 
sociais; renda, posses, condiçôes de vida, ocupação e educação. No entanto, por não serem equivalentes, essas medidas podem ter distintos significados e representar conceitos variados de status social em determinadas culturas (ARAYA et al., 2003).

Estudos de associação entre fatores individuais e contextuais em saúde mental têm sido desenvolvidos no Brasil em diferentes níveis de atenção. O estudo multicêntrico de Gonçalves et al. (2014) avaliou a taxa de transtornos mentais e a associação com variáveis sociodemográficas nas Unidades de Saúde da família. Os autores identificaram que os problemas de saúde mental foram mais elevados altos em mulheres, desempregados e com baixa escolaridade e renda.

Em estudo de prevalência Araya et al. (2001), com 3.870 adultos, encontrou alta associação de transtornos mentais com as variáveis, gênero, desemprego, divórcio, baixo status social e baixo nível educacional. Em outra pesquisa conduzida no Chile (ARAYA et al., 2003), identificou-se uma forte, inversa e estatisticamente significativa associação entre educação e transtornos mentais e relata que esses resultados são similares a outros países da América Latina, embora um estudo britânico tenha encontrado resultados opostos de que a renda e não a educação estejam associadas com os transtornos mentais (ARAYA et al., 2003). Embora os autores apontem que não há clareza sobre a razão para essas divergências, possivelmente essas diferenças de resultados em estudos que usam metodologias semelhantes podem estar relacionadas às questóes culturais, de redes de suporte social e de condiçóes de vida entre diferentes países. Logo, conhecer o impacto dos fatores socioeconômicos na saúde mental requer pesquisa tanto em países desenvolvidos quanto nos países em desenvolvimento, como é o caso do Brasil.

A pesquisa de Maragno et al. (2006), a partir de um inquérito com 2.337 pessoas, identificou transtornos mentais significativamente maiores em mulheres e idosos e com menor renda e escolaridade. Campos, Ramalho e Zanello (2017) destacam que o CAPS é um dos serviços de saúde mental diferenciados na política pública brasileira, pela sua inovação. Em pesquisa, os autores caracterizaram, compararam e analisaram o perfil sociodemográfico de mulheres e homens de um CAPS II. Os resultados apontaram maior presença feminina no serviço e características comuns entre os gêneros, tais como baixa renda e escolarização.

Borba et al. (2017) buscaram conhecer o perfil de 300 usuários de um CAPS no Sul do Brasil. O estudo identificou que 63\% dos usuários eram do sexo feminino, solteiros, com mais de oito anos de escolaridade, desempregados e com renda 
per capita inferior a um salário-mínimo. Os autores justificam que conhecer tais características dos usuários pode auxiliar nas estratégias de intervenção, bem como na qualidade da assistência fornecida. Gomes et al. (2013) encontraram, a partir de pesquisa com 106 usuários de uma Unidade Básica de Saúde do interior paulista, a prevalência de $50 \%$ de transtornos mentais (ansiedade e depressão), além da associação com as variáveis: ocupação, renda, quantidade de medicamentos.

O estudo de Ludermir e Melo-Filho (2002) com 621 adultos a partir de 15 anos registrou prevalência dos transtornos mentais em 35\% da amostra de 62.363 indivíduos, residentes na área II da cidade de Olinda. Nesse estudo a baixa escolaridade $(\mathrm{p}<0,0001)$ e as precárias condiçóes de moradia $(\mathrm{p}=0,02)$ estavam associadas ao desenvolvimento dos transtornos. Os sujeitos com pior situaçáo de renda familiar per capita apresentaram maior prevalência de transtornos mentais.

Pesquisas brasileiras sobre caracterização sociodemográfica e clínica de homens e mulheres, usuários de Centros de Atenção Psicossocial (CAPS II) demonstraram que o baixo nível de escolaridade, baixa renda e a vulnerabilidade social são variáveis relevantes para a análise das condiçóes de vida das pessoas que procuram os serviços de saúde mental. Estes estudos ainda evidenciam uma prevalência de mulheres em atendimento nesses serviços (CAMPOS; RAMALHO; ZANELLO; 2017; CASTRO; COLET, 2011; PEREIRA et al., 2012). Embora a ênfase da presente pesquisa não esteja centrada em sintomas ou diagnósticos dos transtornos mentais, observa-se e destaca-se que esses foram os mais frequentemente relatados durante o momento de acolhimento, por usuários do CAPS II do Distrito Federal, Brasília (CAMPOS; RAMALHO; ZANELLO; 2017), cenário desta pesquisa. Dado o contexto político atual e as mudanças que ameaçam o cuidado em liberdade às pessoas com transtornos mentais (LUSSI et al., 2019), este estudo vem evidenciar a importância da pesquisa sobre o contexto macrossocial que engloba variáveis sociodemográficas e que influenciam na saúde mental da população brasileira, contribuindo para o conhecimento das características dos usuários como forma de melhorar o cuidado e os serviços oferecidos nos âmbitos da prevenção, promoção e reabilitação em saúde.

Frente ao exposto, o propósito deste estudo foi descrever o perfil sociodemográfico de usuários dos serviços de saúde mental de um Centro de Atenção Psicossocial e verificar se existem associaçóes entre a variável escolaridade com as demais variáveis sociodemográficas: idade, gênero, tipo de encaminhamento, estado civil, quantidade de filhos, trabalho, ocupação e renda. 


\section{Materiais e Métodos}

\section{Tipo de pesquisa}

Trata-se de estudo transversal descritivo e associativo do tipo retrospectivo. Este tipo de pesquisa é realizado quando se tem por objetivos descrever e/ou identificar associações entre variáveis, não analisando relações de causa-efeito. Os estudos retrospectivos têm sido adotados como forma de pesquisar características de usuários de serviços de saúde mental (HENNA et al., 2008; SOUSA et al., 2010; GERALDINI et al., 2017; CLEMENTINO et al., 2019).

\section{População}

O presente artigo é a continuidade de uma tese de doutorado desenvolvida no Centro de Atenção Psicossocial (CAPS II), de uma Regiáo Administrativa do Distrito Federal, Brasília, Brasil, que buscou comparar e analisar o perfil sociodemográfico de mulheres e de homens do CAPS II, considerando o recorte no período de 2012 a 2013. Ressalta-se que este CAPS é um dispositivo territorial do Sistema Único de Saúde (SUS) que atende adultos com transtornos mentais graves e persistentes. É constituído por equipe multidisciplinar, composta por psicólogo, enfermeiro, médico psiquiatra, técnico de enfermagem, terapeuta ocupacional e assistente social.

A atenção em saúde mental ofertada pelo CAPS envolve acolhimento, grupos terapêuticos, grupos de trabalho e geração de renda, acompanhamento psiquiátrico, psicológico e social. Tem por objetivo a promoção da cidadania, autonomia e inclusão social, por meio da reabilitação psicossocial. Neste estudo, o período da pesquisa foi de outubro de 2017 a fevereiro de 2018. Optou-se por utilizar a informação sociodemográfica, após a conferência de todas as informaçóes do usuário, considerando-se as últimas atualizações dos prontuários.

\section{Critérios de inclusão e exclusão}

Os critérios de inclusão foram todos os prontuários de usuários ativos admitidos entre os anos de 2014 e 2017. Foram excluídos os prontuários de usuários que não deram continuidade ao tratamento e cujas fichas de acolhimento e/ou evolução psiquiátrica se encontravam no arquivo permanente do serviço; prontuários de usuários que, equivocadamente, encontravam-se no arquivo de ativos, mas que deveriam constar nos arquivos inativos do serviço. 


\section{Coleta de dados}

O estudo teve como fonte da coleta de dados os prontuários de homens e mulheres atendidos CAPS II, a partir das fichas de acolhimento e anamnese psiquiátrica de todos os usuários. O processo de coleta se deu pela impressáo de listas dos pacientes ativos identificados no sistema interno do CAPS e, em seguida, na busca por cada prontuário manualmente, além de consultar as informaçóes de prontuários que poderiam estar em gavetas, e, portanto, não na planilha geral de usuários ativos do serviço. Nessa condição, foram encontrados vinte $(\mathrm{n}=20)$ novos prontuários, sendo que seis $(n=6)$ estavam arquivados e um $(n=1)$ em situação de matriciamento. Nesse sentido, foram incluídos treze $(\mathrm{n}=13)$ prontuários ativos no banco de dados.

A figura 1 descreve o processo de coleta amostral nos prontuários até a definição do número final.

Figuria 1. Procedimentos para a seleçấo dos prontuários (n=488).

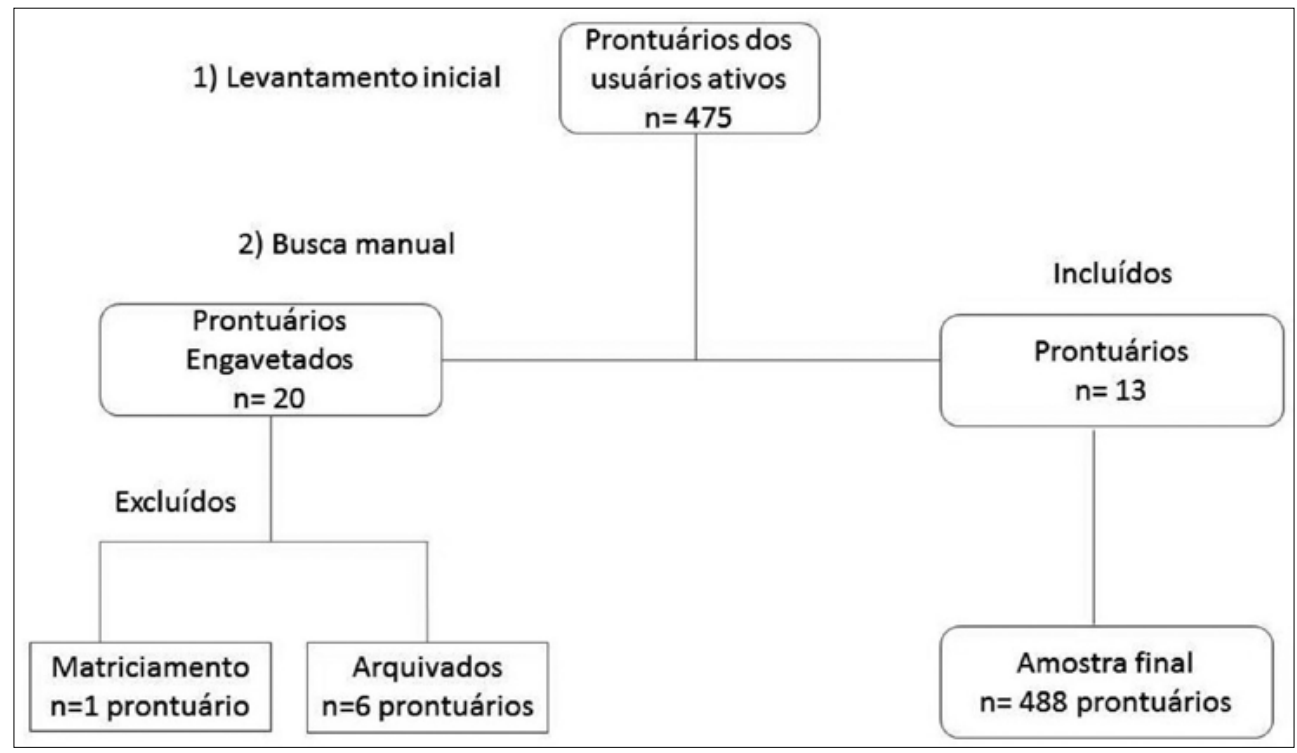

Fonte: Pesquisa de campo.

Posteriormente, todas as fichas foram digitadas diretamente em um banco de dados Microsoft Excel ${ }^{\oplus}$, considerando as seguintes variáveis: idade, sexo; naturalidade, região administrativa, encaminhamento, estado civil, quantidade de filhos, trabalho, escolaridade, ocupação e renda. 


\section{Análise dos dados}

Para a análise dos dados, foi necessário um tratamento inicial dos dados a fim de recategorizar as informaçóes obtidas dos prontuários com fins de análise por relação entre as variáveis. Recategorizaram-se algumas das variáveis, uma vez que, com poucos dados, variáveis com muitas categorias registrariam menores observaçóes em cruzamentos estatísticos. Esta alternativa redistribuiu o contingente entre as categorias e possibilitou obter resultados mais precisos nos testes de associação.

Utilizou-se a análise descritiva por meio de frequências absolutas e percentuais e teste $\chi^{2}$ para a comparação entre as categorias. O nível de significância foi de $95 \%$. Na análise de associação foram utilizados dois testes. Para verificar a associação entre as variáveis nominais, utilizou-se o teste qui-quadrado com simulação de Monte Carlo para o p-valor para correção de amostras pequenas. Para estas variáveis, foi feita também a análise de resíduos padronizados para obter as frequências observadas significativamente acima ou abaixo das frequências esperadas.

Para as variáveis ordinais, foi utilizado um teste não paramétrico para verificação da significância do coeficiente de correlação tau de Kendall, a estatística tau de Kendall varia de -1 a 1 , quando significativa indica o sentido e a força de associação entre as duas variáveis ordinais, valores (em módulo) de 0 a 0,39 foram considerados como associação fraca, de 0,4 a 0,59, associação moderada e de 0,6 a 1 forte. Para ambos os testes, considerou-se um nível de significância de 5\%. Todas as análises foram realizadas no software livre R.

\section{Aspectos éticos}

Este estudo obteve aprovação do Comitê de Ética em Pesquisa da Faculdade de Ceilândia e da Fundação de Ensino e Pesquisa em Ciências da Saúde/FEPECS/SES/ DF, sob o parecer de número 2.586.152, CAAE número 77679817.9.0000.8093.

\section{Resultados}

Dos 488 prontuários consultados, 324 eram de mulheres e 164 homens, admitidos entre os anos de 2014 e 2017. A tabela 1, a seguir, apresenta os dados descritivos de recategorização das variáveis consideradas para análise: 
Tabela 1. Valores absolutos e relativos por variável e sexo e teste qui-quadrado para homogeneidade das categorias. Brasília - Distrito Federal, 2018.

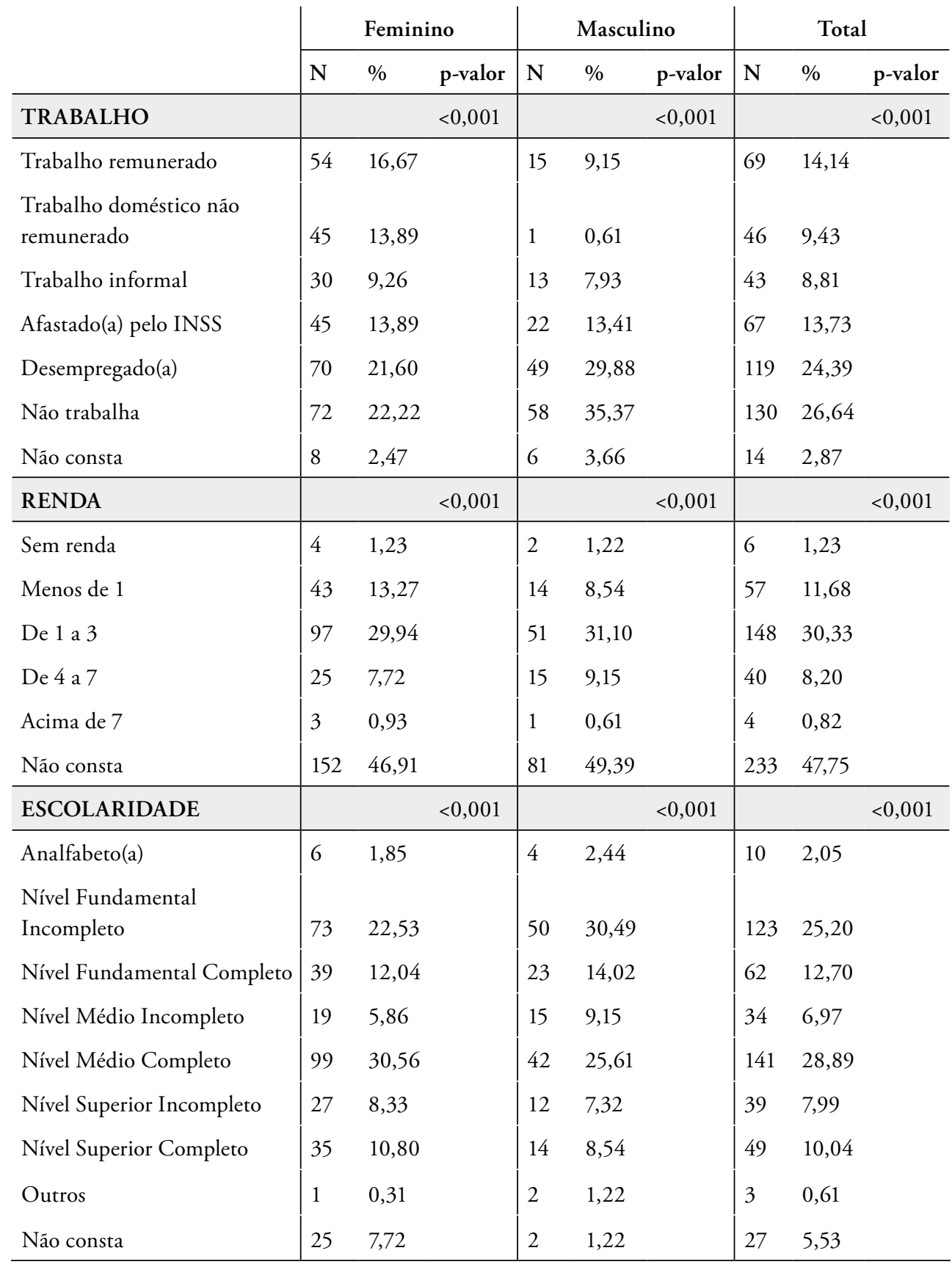




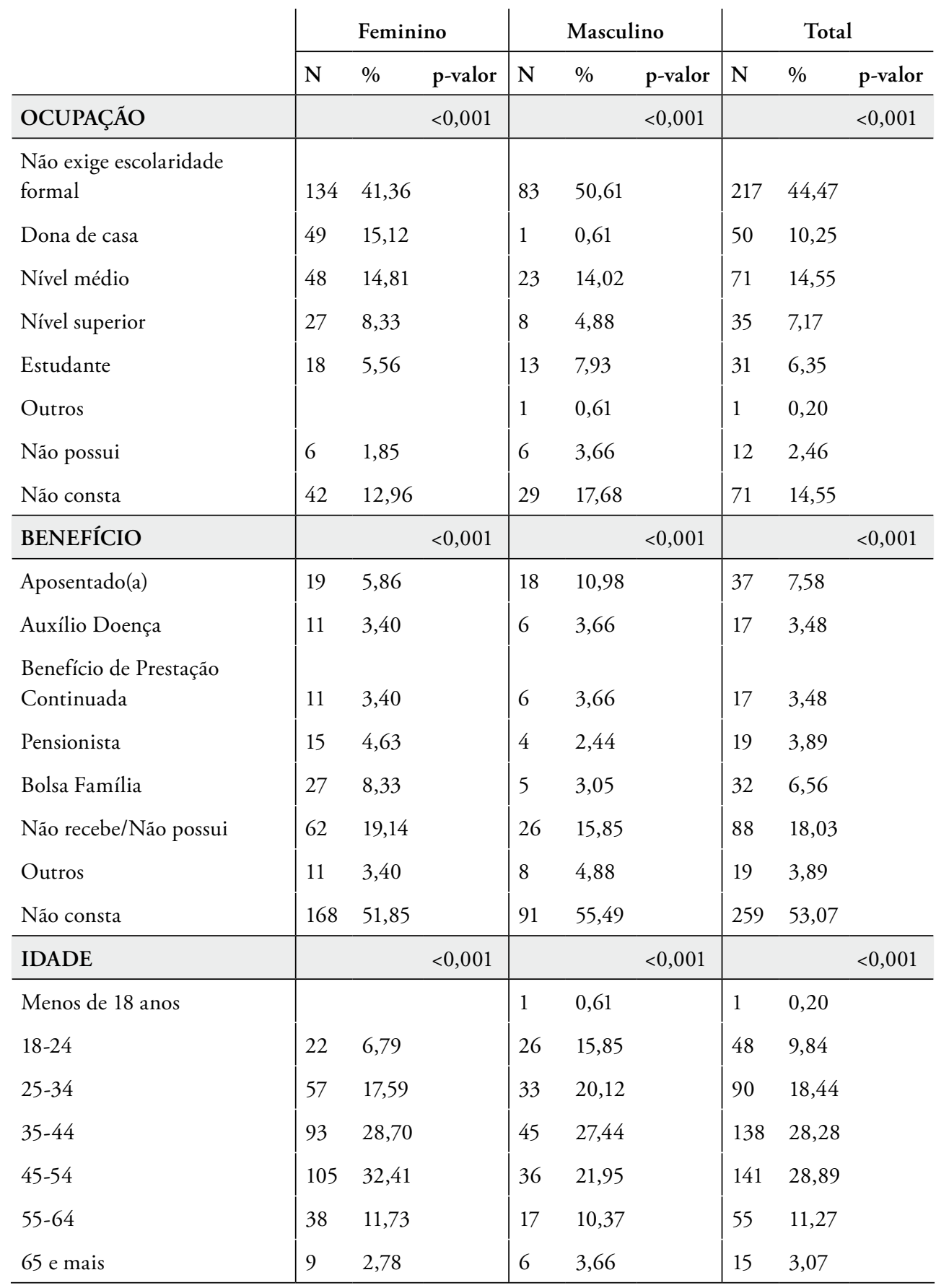




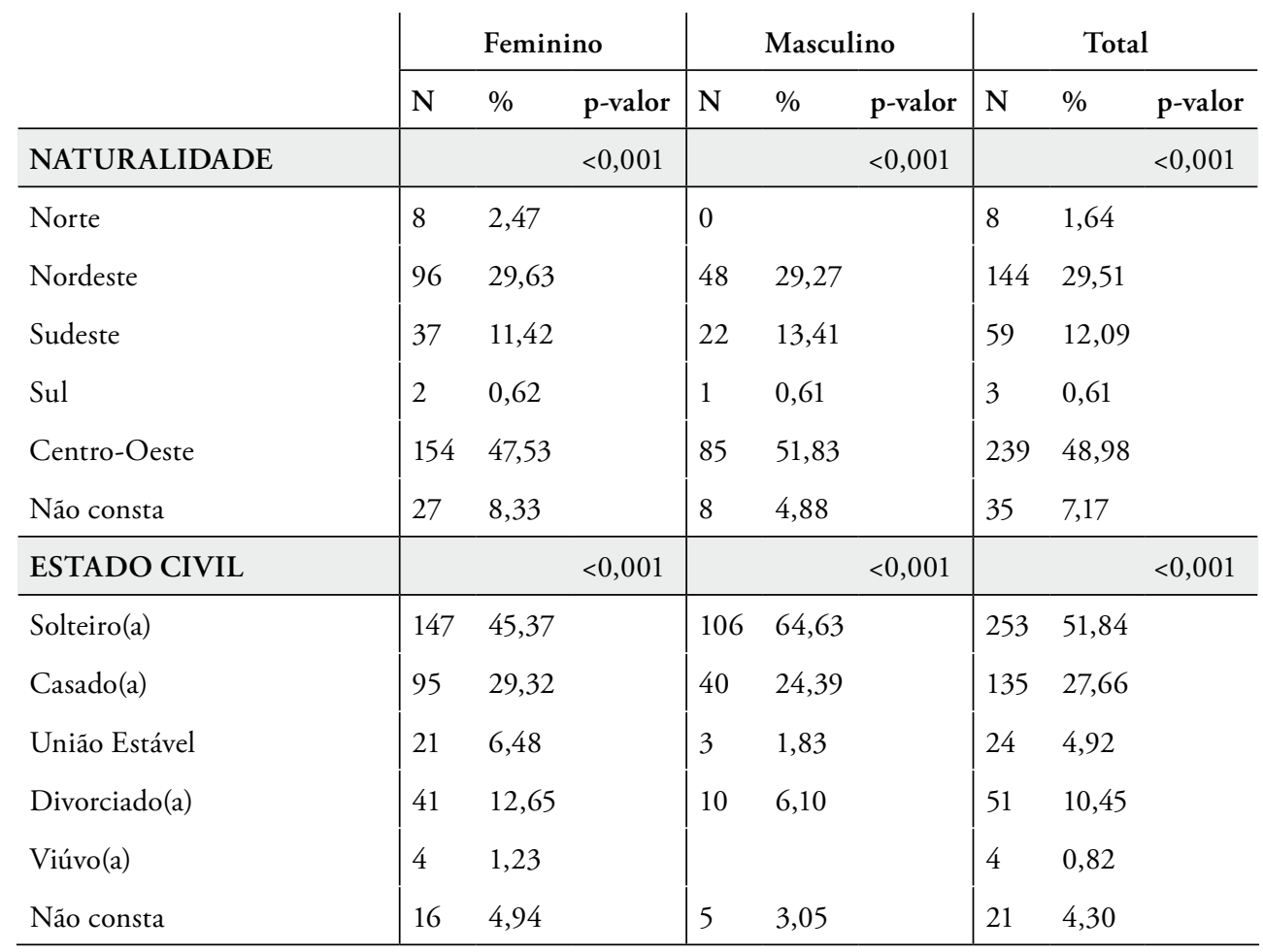

Cabe esclarecer que dois dados da tabela 1 (renda e receber benefício) tiveram maior porcentagem de informaçóes faltantes, com respectivamente $46,91 \%$ e 51,85 identificados pelo item "não consta". Os dados descritivos demonstram que, quando comparadas as frequências encontradas para homens e mulheres, verificou-se uma quantidade predominante para homens em trabalho informal com até ensino médio completo; já para as mulheres, o ensino superior completo com trabalho remunerado.

Houve associação significativa entre ocupação e escolaridade, com frequência acima da esperada para as seguintes combinaçóes de categoria: dona de casa analfabeta e com escolaridade até ensino fundamental completo; estudante e ocupação; que não exige escolaridade formal com escolaridade até ensino fundamental completo; ocupação que exige nível médio com ensino médio completo e; ocupação que exige nível superior completo com ensino superior completo.

Em menor frequência, foram observadas as seguintes categorias: ocupaçôes que exigem ensino médio com escolaridade até ensino fundamental completo; ocupaçôes que não exigem escolaridade formal e que exigem superior completo com ensino 
médio completo e; dona de casa, estudante, ocupações que não exigem escolaridade formal e que exigem nível médio com escolaridade de ensino superior completo.

Buscou-se identificar se havia diferenças entre os níveis de escolaridade para as mulheres e os homens. Porém, no cruzamento entre gênero e escolaridade utilizando o teste qui-quadrado, não foram encontradas diferenças significativas $(\mathrm{p}=0.14)$. A tabela 2 apresenta os resultados dos testes de relação entre escolaridade e variáveis ocupação, renda, quantidade de filhos e benefício:

Tabela 2. Associação entre escolaridade e variáveis trabalho, ocupaçáo, renda, quantidade de filhos e benefício (n=488). Brasília-Distrito Federal, 2018.

\begin{tabular}{|c|c|c|c|c|c|}
\hline Escolaridade & $\begin{array}{l}\text { Analfabeto } \\
\text { (a) }\end{array}$ & $\begin{array}{l}\text { Até ensino } \\
\text { fundamental } \\
\text { completo }\end{array}$ & $\begin{array}{l}\text { Ensino } \\
\text { médio } \\
\text { completo }\end{array}$ & $\begin{array}{l}\text { Ensino } \\
\text { superior } \\
\text { completo }\end{array}$ & $\mathrm{p}$-valor \\
\hline Trabalho & & & & & 0,00050 \\
\hline Afastado pelo INSS & 1 & 23 & 27 & 13 & \\
\hline Desempregado/Não trabalha & 2 & 120 & 95 & 17 & \\
\hline Trabalho doméstico não remunerado & 4 & 22 & 18 & 1 & \\
\hline Trabalho informal & 1 & 22 & 14 & 3 & \\
\hline Trabalho remunerado & 0 & 22 & 26 & 15 & \\
\hline Ocupação & & & & & 0,00050 \\
\hline Dona de casa & 3 & 29 & 15 & 1 & \\
\hline Estudante & 0 & 12 & 19 & 0 & \\
\hline Não exige escolaridade formal & 3 & 125 & 70 & 8 & \\
\hline Nível médio & 0 & 6 & 4 & 1 & \\
\hline Nível Superior & 1 & 12 & 46 & 6 & \\
\hline Não possui & 0 & 1 & 2 & 32 & \\
\hline Renda (SM) & & & & & $<0,0001^{*}$ \\
\hline Sem Renda & 0 & 0 & 3 & 1 & \\
\hline Menos de 1 & 4 & 73 & 58 & 8 & \\
\hline De 1 a 3 & 0 & 13 & 16 & 9 & \\
\hline De 4 a 7 & 2 & 33 & 13 & 2 & \\
\hline Acima de 7 & 4 & 97 & 88 & 29 & \\
\hline
\end{tabular}




\begin{tabular}{l|ccccc}
\hline Escolaridade & $\begin{array}{l}\text { Analfabeto } \\
\text { (a) }\end{array}$ & $\begin{array}{l}\text { Até ensino } \\
\text { fundamental } \\
\text { completo }\end{array}$ & $\begin{array}{l}\text { Ensino } \\
\text { médio } \\
\text { completo }\end{array}$ & $\begin{array}{l}\text { Ensino } \\
\text { superior } \\
\text { completo }\end{array}$ & p-valor \\
\hline Quantidade de filhos & 1 & 44 & 35 & 10 & $\mathbf{0 , 0 0 7 9 0 ^ { * }}$ \\
\hline 0 & 1 & 29 & 26 & 10 & \\
1 & 1 & 34 & 34 & 12 & \\
2 & 1 & 31 & 21 & 5 & \\
3 & 0 & 20 & 7 & 0 & \\
4 & 3 & 14 & 6 & 0 & \\
Acima de 4 & & & & & \\
\hline Benefício & 5 & 71 & 41 & 10 & \\
\hline Recebe & 0 & 35 & 37 & 12 & \\
Náo possui/Náo recebe & & & & & \\
\hline
\end{tabular}

*Teste tau de Kendall

Os testes de associação entre escolaridade e as outras variáveis de estudo indicaram associação significativa para trabalho $(\mathrm{p}=0.0005)$, ocupação $(\mathrm{p}=0.0005)$, renda $(\mathrm{p}<0.0001)$, quantidade de filhos $(\mathrm{p}<0.007)$ e receber benefício $(\mathrm{p}=0.02)$. Para a associação entre escolaridade e trabalho, verificou-se frequência acima da esperada para o cruzamento das categorias: trabalho doméstico não remunerado com analfabeto(a); desempregado(a) / não trabalha com escolaridade até ensino fundamental completo e; afastado pelo INSS com ensino superior completo. Atenta-se também para as frequências abaixo das esperadas: trabalho remunerado com escolaridade até ensino fundamental completo; desempregado/não trabalha com ensino superior completo; trabalho doméstico não remunerado com ensino superior completo.

Associação significativa, porém, fraca, foi identificada entre renda e escolaridade, em que quanto maior a escolaridade maior a renda. Também foi identificada a tendência associativa inversamente proporcional na qual quanto maior a escolaridade, menor a quantidade de filhos; no entanto, tal associação, embora estatisticamente significativa, foi fraca.

Por fim, foi observada associação entre receber benefício e nível de escolaridade, com significância para as seguintes relaçôes: receber benefício e ter até o ensino fundamental completo; e com baixa frequência para quem não recebe benefício com a categoria de escolaridade até ensino fundamental completo. 


\section{Discussão}

O presente estudo buscou identificar associaçôes entre variáveis sociodemográficas de usuários de um serviço de saúde mental. Os dados encontrados permitem discutir os aspectos sociodemográficos dos usuários de um serviço de saúde mental e sua condição de saúde. De acordo com Maragno et al. (2006), pelo fato de os transtornos mentais se apresentarem relacionados a indicadores de desvantagem social, há a urgência de atenção especial focalizada nos problemas e grupos de risco específicos.

Especificamente sobre o baixo nível de escolaridade encontrado em nosso estudo, pode-se discutir que este pode ser um dos fatores determinantes para que os trabalhadores com menor oportunidade de estudo sejam obrigados a se inserir no mercado informal de trabalho (PATEL; KLEINMAN, 2003). O nível de escolaridade é uma das variáveis que pode influenciar melhores condições de vida aos indivíduos, sendo uma delas a inserção no mercado de trabalho (LUDERMIR, 2008; LUDERMIR; MELO FILHO, 2002).

Nossa pesquisa identificou, a partir dos dados descritivos, trabalhos informais exercidos por 40 usuários e que, portanto, não exigem qualificação, em termos de estudo. Dado similar foi relatado na pesquisa de Araya et al. (2003), em que se identificou que a prevalência de transtornos mentais em chilenos foi maior para aqueles inseridos em ocupaçóes que não exigiam qualificação, e que apresentavam baixa renda per capta.

Cabe discutir que a inserção de usuários de serviços de saúde mental no trabalho é importante forma de inclusão dessas pessoas, envolvendo trocas sociais fundamentais ao indivíduo na sociedade. Essa inclusão pelo trabalho, entretanto, não parece ser uma realidade no Brasil. Em estudo conduzido por Paranhos-Passos e Aires (2013), com 20 usuários de saúde mental de um CAPS, identificou-se que nenhum deles encontrava-se trabalhando. As autoras concluíram que a reinserção social dessas pessoas ainda não havia alcançado outros espaços para além do CAPS.

Em relação ao nível de ocupação, este é quase sempre crescente em relação à escolaridade. Isso significa que quanto maior a escolaridade, maior a proporção de pessoas ocupadas em relação à população com menor escolaridade. Nosso estudo verificou essa tendência. Pode-se discutir que completar um ciclo educacional sempre resulta em um nível de ocupaçáo maior que o mesmo nível incompleto. E que, enquanto a maior parte da população tem o ensino fundamental incompleto 
(49 milhões) ou ensino médio completo (45 milhōes), o maior nível de ocupação está dentre aqueles que têm o ensino superior (77\%) (BARCELLOS, 2019).

A falta de estudo e ou dificuldades de acesso à escola, trabalho e as condiçóes socioeconômicas podem ser fatores de risco para problemas de saúde mental ou mesmo em seu agravamento, na medida em que influenciam a autonomia do indivíduo e sua capacidade reflexiva e de participar de trocas sociais (CAMPOS; RAMALHO, ZANELLO). Os dados encontrados em nosso estudo sobre baixa escolaridade dos usuários do CAPS corroboram os achados de Campos, Ramalho e Zanello (2017) em pesquisa com 234 usuários de um serviço de saúde mental. Os autores identificaram que $48,7 \%$ dos usuários eram analfabetos ou possuíam o ensino fundamental, sendo que apenas $8,5 \%$ tinham ensino superior completo.

Em estudo recente, Campos e Magalhães (2019) entrevistaram três pessoas analfabetas, usuárias de um CAPS. As autoras identificaram um histórico de trabalho precoce que interrompeu a permanência na escola. Foram relatadas a ausência de suporte social e familiar, fatores socioeconômicos e o estigma (preconceito) frente ao adoecimento mental. Este último parece acompanhar a trajetória de vida de pessoas com transtornos mentais na sociedade. Sanches e Oliveira (2011) relatam um estudo de dois casos a partir do relato de pais de usuários de serviços de saúde mental com relaçáo à sua inclusão escolar; da escola especial para o ensino regular em um município de São Paulo, e referem que as mães sentem falta de discussões intersetoriais, a fim de que o sofrimento de seus filhos possa ser minimizado, ampliando o contexto social de inclusão.

Pelo fato de as variáveis trabalho e educação terem sido associadas em nosso estudo como relevantes nos usuários investigados, estes podem ser identificados como elementos centrais na questão socioeconômica, estando intrinsecamente relacionados. Balassiano, Seabra e Lemos (2005) estudaram a relação envolvendo escolaridade, salário e empregabilidade a partir de dados da Relação Anual de Informaçôes Sociais (RAIS) na região metropolitana do Rio de Janeiro, para o ano de 1999. Como resultados, identificaram que há um impacto positivo do aumento da escolaridade na renda do trabalhador, mas o efeito é significativo apenas para as faixas etárias de maior escolaridade, a partir do segundo grau. Então, o aumento da escolaridade resulta em incremento da renda apenas aos que possuem maior escolaridade (BALASSIANO; SEABRA; LEMOS, 2005). 
Um estudo sobre escolaridade e desigualdade de renda identificou que quanto maior o percentual de escolaridade consequentemente maior será a renda; também destacou, porém, a existência de outros fatores, como de vida dos habitantes, fatores étnicos, estrutura etária da população, qualidade da infraestrutura existente, presença/ausência de estímulos ao desenvolvimento e fatores históricos, que afetam diretamente a variável renda entre os indivíduos (SALVATO; FERREIRA; DUARTE, 2010). Souza et al. (2014) investigaram a relação entre desigualdade de renda e educação no Brasil e apresentaram que os resultados obtidos estão de acordo com estudos anteriores em relação às variáveis renda e educação como inversamente relacionadas com a desigualdade de renda.

Em outro estudo, Araya et al. (2003) avaliaram indicadores socioeconômicos com um aumento da prevalência de transtornos mentais. A partir de amostra randomizada de adultos entre 16-65 anos, em Santiago, Chile, os autores reportaram que pobre moradia, baixo nível educacional e redução da renda estiveram associados significativamente com o aumento da prevalência desses tipos de transtornos mentais.

De acordo com nossos achados, as duas variáveis escolaridade e quantidade de filhos também apresentaram associação, onde quanto maior o nível de escolaridade, menor a quantidade de filhos. Lopes e Pontilli (2008) relatam que várias pesquisas relacionam que um maior nível educacional e renda parecem ter influência negativa quanto ao desejo de fertilidade. Tais estudos têm sustentado que o aumento da escolaridade é positivo nas chances de trabalho e nos salários. Segundo dados do IBGE (2010), mulheres com menor escolaridade têm maior número de filhos, no Brasil. Isso concorda com as constataçóes de estudos anteriores (DE MARIA, 2014; LOPES; PONTILLI, 2008).

Esses estudos estão em consenso que, conforme aumenta o nível de escolaridade da mulher, diminui a quantidade de filhos desejados, ou seja, quanto maior o nível de instrução menor a probabilidade de que elas tenham um número elevado de filhos. $\mathrm{O}$ mesmo estudo afirma também que, quanto maior o nível de instrução das mulheres, mais fácil sua inserção no mercado de trabalho e, portanto, menor o número de filhos que estas desejarão ter (DE MARIA, 2014; LOPES; PONTILLI, 2008). Por outro lado, cabe discutir que existem evidências recentes de que o número de filhos tem associação com o poder aquisitivo (BARBANO; CRUZ, 2019). 
Quanto à escolaridade-benefício, corroborando nossos achados, um estudo verificou associaçáo da escolaridade e a utilizaçáo de benefícios, observando que famílias que recebem benefícios apresentam baixa escolaridade (DEDECCA et al., 2006).

Em países em desenvolvimento, o baixo nível de educação é o indicador de pobreza mais consistentemente associado ao risco de transtorno mental (PATEL; KLEINMAN, 2003). Pelos resultados apresentados em nosso estudo, pode-se discutir que na amostra estudada, os usuários com transtornos mentais apresentaram associaçóes quanto a variáveis sociodemográficas que, de certa forma relacionam a pobreza aos problemas de saúde mental. Cabe discutir que esta afirmação considera um conceito de pobreza ampliado e não restrito apenas à variável renda. Dessa forma, pobreza do ponto de vista epidemiológico está relacionada a um baixo nível socioeconômico, o que inclui baixo rendimento, desemprego, nível de escolaridade inferior e baixa posição da família na hierarquia social (SILVA; CARDOSO; ALMEIDA, 2015).

Reforçam esta discussão estudos sobre a prevalência de transtornos mentais, os quais apresentaram que os mesmos estão associados às variáveis relativas às condiçôes de vida, analfabetismo, baixa escolaridade, precariedade de moradia, o médio acesso aos bens de consumo duráveis (LUDERMIR; MELO FILHO, 2002; MARAGNO et al., 2006). Sustentam nossos resultados outros estudos que identificaram que a baixa escolaridade está relacionada com uma maior prevalência de transtornos psiquiátricos (ARAYA et al., 2001; LIMA; BERIA; TOMASI, 1996). Dessa forma, a educação é uma das variáveis importantes na associação com a pobreza e transtornos mentais, sendo um indicador sensível para informar a carência de oportunidades ou baixa posição social em países com grandes desigualdades socioeconômicas (ARAYA et al., 2001; 2003).

Desse modo, os dados revelados por nosso estudo apontam uma pertinência da intersetorialidade de serviços de assistência em saúde mental a partir de uma visão abrangente dos aspectos que interferem na saúde dessas pessoas nos âmbitos teóricos, políticos e culturais, a fim de que a reinserção dessas pessoas possa estar na pauta e nos objetivos de facilitar a reinserção social a partir da atenção psicossocial (CAMPOS; MAGALHÁES, 2019).

\section{Considerações finais}

O presente estudo identificou associaçóes relevantes sobre a variável escolaridade de usuários de serviços de saúde mental de um CAPS II, tais como trabalho, ocupação, 
renda, quantidade de filhos e receber benefício. Esses dados podem fornecer subsídios importantes para a discussão sobre educação, trabalho e renda como variáveis pertinentes para uma discussão ampliada sobre saúde mental para além de diagnósticos e trazer reflexôes para ações de assistência a esses grupos, incluindo as preventivas.

Cabe esclarecer que este estudo apresenta limitaçôes. Uma delas é a de que a análise de associação de escolaridade com as outras variáveis, quando separada por sexo, teve sua precisão de estimativas comprometida, uma vez que o tamanho da amostra foi pequeno para emitir conclusóes. $\mathrm{Na}$ análise de resíduos do teste quiquadrado, não foi feita nenhuma correção para tamanho de amostras, portanto, as aproximaçóes do qui-quadrado realizadas pelo software podem apresentar vieses pelos mesmos motivos.

Neste estudo, também emergiram dificuldades para levantamento sociodemográfico, sobretudo decorrentes de letras ilegíveis nos prontuários, dados incompletos e vários campos sem preenchimento (renda familiar, ocupação, benefícios sociais e/ou de saúde recebidos e exame psíquico), o que limitou os achados e o aprofundamento das análises estatísticas. Por outro lado, os resultados encontrados com estas análises são representativos para a amostra estudada, podendo gerar hipóteses para futuros estudos - por exemplo, para investigar o perfil de mulheres e homens no CAPS, em especial a correlação entre saúde mental, vulnerabilidade e formação escolar básica.

Em conclusão, a importância de pesquisas que investiguem correlaçóes entre dados sociodemográficos e saúde mental fornecem evidências de base para a criação e/ou incremento de políticas públicas nesse campo. Para tanto, há a necessidade de um trabalho colaborativo entre os diversos setores de saúde mental e a educação por meio das ações intersetoriais que contribuam a para inclusão do usuário na formação escolar básica e, consequentemente, em seus desdobramentos e possibilidades, tais como empregabilidade, não dependência de benefício e constituição familiar. Isto requer também considerar as singularidades e histórico de vida dos usuários para que sejam formuladas metodologias de processos educativos para essa população. ${ }^{1}$

\section{Agradecimentos}

À Fundação de Apoio à Pesquisa do Distrito Federal (FAPDF), pelo financiamento; e aos alunos do curso de graduação em Psicologia da Universidade de Brasília e 
apoio técnico: Bernardo Geraldo Domingos, Ezequiel Ruiz dos Santos, Gabrielle Krystine Virginia Rodrigues Silva e Lívia Barbosa Alves de Souza, que participaram como bolsistas do projeto "Educação Inclusiva e Saúde Mental: a construção de um diagnóstico territorial em uma capital brasileira".

\section{Referências}

ARAYA, R. et al. Education and income: which is more important for mental health? Journal of Epidemiology \& Community Health, v. 57, n. 7, p. 501-505, jul. 2003.

ARAYA, R. et al. Common mental disorders in Santiago, Chile: prevalence and sociodemographic correlates. Br. J. Psychiatry, v. 178, n. 3, p. 228-33, mar. 2001.

BARBANO, L. M.; CRUZ, D. M. C. Time use, purchasing power, and job satisfaction: Correlation and comparison between working women with children and working women without children. Work, v. 62, n. 4, p. 563-71, maio 2019.

BALlARIN, M. L. G. S.; MIRANDA, I. M. S.; FUENTES, Ana Carolina de Ramos Castelhano. Centro de atenção psicossocial: panorama das publicaçóes de 1997 a 2008. Psicol. Cienc. Prof., v. 30, n. 4, p. 726-737, dez. 2010.

BALASSIANO, M.; SEABRA, A. A.; LEMOS, A. H. Escolaridade, salários e empregabilidade: tem razão a teoria do capital humano? Rev. Adm. Contemp., v. 9, n. 4, p. 31-52, dez. 2005.

BARCELLOS, T. Como está o nivvel de ocupação por escolaridade? Disponível em: <https://idados. id/blog/como-esta-o-nivel-de-ocupacao-por-escolaridade>. Acesso em: 18 ago. 2019.

BORBA, L. O. et al. Perfil do portador de transtorno mental em tratamento no Centro de Atenção Psicossocial (CAPS). REME - Rev. Min. Enferm., v. 21, p. e1069, dez. 2017.

BRASIL. Ministério da Saúde. Secretaria de Atenção à Saúde. Departamento de Atenção Básica. Saúde mental. Brasília: Ministério da Saúde, 2013.

CAMPOS, I. O.; MAGALHÁES, Y. B. Analfabetismo e Saúde Mental: O Discurso de Usuários de um Centro de Atenção Psicossocial. Rev. FSA, v. 16, n. 4, p. 151-65, jul-ago. 2019.

CAMPOS, I.O.; RAMALHO, W.M.; ZANELLO, V. Saúde mental e gênero: O perfil sociodemográfico de pacientes em um centro de atenção psicossocial. Estud. Psicol., v. 22, n. 1, p. 68-77, mar. 2017.

CASTRO, A. L.; COLET, C. Perfil socioeconômico e características da depressão de usuários do Centro de Atenção Psicossocial (CAPS) de Panambi-RS. Rev. Contexto \& Saúde, v. 11, n. 20, p. 401-408, jan-jun. 2011.

CLEMENTINO, F. S. et al. Atendimento integral e comunitário em saúde mental: avanços e desafios da reforma psiquiátrica. Trab., Educ. e Saúde, v. 17, n. 1, fev. 2019. 
DE MARIA, P. F. A transição da fecundidade e da escolaridade nas famílias pobres brasileiras: evidências a partir das PNADs 1978 a 2012. Anais. Trabalho apresentado no XIX Encontro Nacional de Estudos Populacionais, ABEP, realizado em São Pedro-SP, Brasil, 24-28 nove. 2014.

DEDECCA, C. S. et al. Salário-mínimo, benefício previdenciário e as famílias de baixa renda. Rev. Bras. Estud. popul., v. 23, n. 2, p. 317-329, dez. 2006.

GERALDINI, A. et al. Ambulatório médico de especialidades-psiquiatria Vila Maria: balanço dos dois anos iniciais. Psic., Saúde \& Doenças, v. 18, n. 2, p. 503-511, 2017.

GONÇALVES, D. A. et al.Brazilian multicentre study of common mental disorders in primary care: rates and related social and demographic factors. Cadernos de Saúde Pública, v. 30, n. 3, p. 623-632, 2014.

GOMES, V. F. et al. Transtornos mentais: perfil sociodemográfico e farmacoterapêutico. Rev. Latino-Am. de Enf., v. 21, n. 6, p. 1203-11, nov-dez. 2013.

HENNA, E. S. et al. Rede de Atenção à Saúde Mental de Base Comunitária: a experiência de Santo André. Journal of Human Growth and Development, v. 18, n. 1, p. 16-26, abr. 2008.

LOPES, J. L.; PONTILLI, R. M. Renda familiar e educação como fatores condicionantes do aumento da taxa de fertilidade: uma análise para a regiáo sul do Brasil. Cad. de Econ., v. 14., n. 27, p. 25-35, jul-dez. 2008.

LIMA, M. S. et al. Stressful life events and minor psychiatric disorders: An estimate of the population attributable fraction in a Brazilian community-based study. International Journal of Psychiatry in Medicine, v. 26, n. 2, p. 211-222, 1996.

LUDERMIR, A. B. Desigualdades de classe e gênero e saúde mental nas cidades. Physis: Revista de Saúde Coletiva. Rio de Janeiro, v. 18, n. 3, p. 451-467, set. 2008.

LUDERMIR, A. B.; DE MELO FILHO, D. A. Condiçóes de vida e estrutura ocupacional associadas a transtornos mentais. Rev. de Saúde Públ., v. 36, n. 2, p. 213-221, abr. 2002.

LUSSI, I. A. O. et al. Saúde mental em pauta: afirmação do cuidado em liberdade e resistência aos retrocessos. Cad. Bras. Ter. Ocup., v. 27, n. 1, p. 1-3, jan. 2019.

MARAGNO, L. et al. Prevalência de transtornos mentais em populaçóes atendidas pelo Programa Saúde da Família (QUALIS) no Município de São Paulo, Brasil. Cad. Saúde Pública, v. 22, n. 8, p. 1639-48, ago. 2006.

PARANHOS-PASSOS, F.; AIRES, S. Reinserçáo social de portadores de sofrimento psíquico: o olhar de usuários de um Centro de Atenção Psicossocial. Physis: Revista de Saúde Coletiva. Rio de Janeiro, v. 23, n. 1, p. 13-31, 2013.

PATEL, V.; KLEINMAN, A. Poverty and common mental disorders in developing countries. Bulletin of the World Health Organization, v. 81, n. 8, p. 609-15, out. 2003. 
PEREIRA, M. O. et al. Perfil dos usuários de serviços de Saúde Mental do município de Lorena - São Paulo. Acta Paul. Enferm., v. 25, n. 1, p. 48-45, 2012.

PITTA, A. M. F. Um balanço da reforma psiquiátrica brasileira: instituições, atores e políticas. Ciênc. e Saúde Col., v. 16, n. 12, p. 4579-89, dez. 2010.

SANCHES, A. C. G.; OLIVEIRA, M. A. F. Educação inclusiva e alunos com transtorno mental: um desafio interdisciplinar. Psic.: Teor. e Pesq., v. 27, n. 4, p. 411-18, dez. 2011.

SALVATO, M. A.; FERREIRA, P. C. G.; DUARTE, A. J. M. O impacto da escolaridade sobre a distribuição de renda. Estud. Econ., v. 40, n. 4, p. 753-791, dez. 2010.

SARACENO, B. Libertando identidades: da reabilitaçâo psicossocial à cidadania possivel. Rio de Janeiro: Instituto Franco Basaglia; Te Corá, 1999.

SILVA, M. et al. Saúde mental e a crise econômica. In: SANTANA, P. (Coord.). Território e Saúde Mental em Tempos de Crise. Coimbra: Pombalina, Imprensa da Universidade de Coimbra, 2015, p.61-74.

SOUSA, F. S. P.; SILVA, C. A. F.; OLIVEIRA, E. N. Serviço de Emergência Psiquiátrica em Hospital Geral: estudo retrospectivo. Rev. da Escola de Enf. da USP, v. 44, n. 3, p. 796-802, set. 2010 .

SOUZA, M. I. A. et al. Relação entre a desigualdade e educação no Brasil: uma estimativa de dados. In: IV Semana do Economista e IV Encontro de Egressos. Anais. Universidade Estadual de Santa Cruz - UESC, Ilhéus-BA, 7-10 out. 2014.

\section{Nota}

${ }^{1}$ I. O. Campos e D. S. Rodrigues: elaboração, sistematização, redação e revisão final. D. M. C da Cruz: sistematização e revisão final. Y. B. Magalhães: elaboração, redação e revisão final. 


\section{Abstract}

Schooling, work, income and mental health: a retrospective and association study with users of a psychosocial care center

Sociodemographic studies are important for planning and analyzing the needs of the population. Research on the profile of users of mental health services is still incipient in Brazil. We aimed to describe the sociodemographic profile of users of a psychosocial care center and to verify if there are associations between the variable education with the other demographic variables. This is a cross-sectional, descriptive, associative, and retrospective study, with data gathered through by medical records, protocol of reception, social report and psychiatric anamnesis. Data analysis was performed through a descriptive and association tests. The prevalence of women users in the services was identified $(\mathrm{n}=324)$. The users came mainly from the Middle-West $(n=239)$ of the country, followed by the Northeast $(\mathrm{n}=144)$. The social conditions stated a high number of unemployment $(\mathrm{n}=130)$, in addition to the elementary and high school levels. The variables: work $(\mathrm{p}=$ $0.0005)$, occupation ( $p=0.0005)$, income ( $<<0.0001)$, number of children $(\mathrm{p}<0.007)$ and receive benefit $(\mathrm{p}$ $=0.02$ ) presented positive correlations. We concluded on the importance of research of correlations between sociodemographic data and mental health variables to provide evidence in the creation of public policies in this field and social inclusion of users.

> Keywords: mental health; social conditions; education; work; rehabilitation. 